\title{
ARTICLE
}

\section{Zymosan by-passes the requirement for pulmonary antigen encounter in lung tissue-resident memory $\mathrm{CD}^{+} \mathrm{T}$ cell development}

\author{
Irina Caminschi ${ }^{1}$, Mireille H. Lahoud ${ }^{1}$, Angela Pizzolla ${ }^{2}$ and Linda M. Wakim ${ }^{2}$
}

\begin{abstract}
Tissue-resident memory T cells (Trm) in the lung provide a frontline defence against respiratory pathogens. Vaccination models that lodge $\mathrm{CD}^{+}$Trm populations in the lung have been developed, all of which incorporate the local delivery of antigen plus adjuvant into the airways; a necessary approach as local cognate antigen recognition is required for optimal lung Trm development. Although pulmonary delivery of antigen is important for lung Trm development, the impact the co-administered adjuvant has on Trm differentiation is unclear. We show that while altering the adjuvant co-administered with the pulmonary delivered antigen does not impact the size of the lung Trm population, a particular adjuvant, zymosan, when administered into the airways without antigen can drive effector $\mathrm{CD}^{+}{ }^{\mathrm{T}}$ cells to differentiate into lung Trm. Zymosan signalling via dectin-1 receptor was sufficient to promote antigen-independent lung Trm development. When combined with an injectable influenza vaccination regime, intranasal zymosan delivery significantly boosted the size of the influenza virus-specific lung Trm population. Our results highlight that eliciting the appropriate local inflammatory milieu can by-pass the requirement for local antigen recognition in lung Trm development and emphasises that the appropriate selection of adjuvant can greatly improve vaccines that aim to elicit pulmonary Trm.
\end{abstract}

Mucosal Immunology (2019) 12:403-412; https://doi.org/10.1038/s41385-018-0124-2

\section{INTRODUCTION}

The respiratory mucosa is in direct contact with the environment and is continuously exposed to airborne microorganisms, as such, it is a major site for pathogen invasion. Vaccines that safeguard this mucosal tissue will curb respiratory infections, which to date continue to be one of the leading causes of morbidity and mortality worldwide. Tissue-resident memory T cells (Trm) in the lung provide a frontline defence against respiratory pathogens and lodging Trm along the respiratory tract has proven to be an effective approach to limit the severity of infection mediated by a range of respiratory pathogens. ${ }^{1-7}$

$\mathrm{CD}^{+}$Trm cells are a non-circulating, self-renewing subset of memory $\mathrm{T}$ cells that reside permanently in a variety of barrier tissues, including the respiratory tract. ${ }^{8}$ Trm have a unique transcriptional programme $e^{9-12}$ that promotes long-term tissue residency and survival; key features include the downregulation of KLF2, ${ }^{13}$ T-bet and Eomes ${ }^{14}$ and the upregulation of Hobit and Blimp-1, ${ }^{15}$ all of which drive the upregulation of proteins important in tissue retention (ie, CD103, CD69, CD49a, CXCR6) and the downregulation of proteins that favour T-cell egress (S1P1R, CD62L, CX3CR1). Trm confer rapid and robust local immune protection both directly, by killing pathogen infected cells and releasing cytokines that render the local environment non permissive for pathogen replication, ${ }^{16}$ and indirectly, by promoting the recruitment of other immune cells from the circulation into the site of infection. ${ }^{17}$ When deposited along the respiratory tract, Trm provide better protection against inhaled pathogens than their circulating memory T-cell counterparts. ${ }^{1-7}$ In the case of influenza virus infection, pulmonary Trm are indispensable at providing heterosubtypic immunity ${ }^{2}$ and can limit the movement of influenza virus from the upper to the lower respiratory tract, ${ }^{1}$ a situation associated with severe disease. Vaccination strategies that deposit Trm along the respiratory tract are likely to confer long-lasting protection against inhaled pathogens. A critical step in the development of a vaccine that evokes pulmonary Trm is defining the parameters that promote Trm formation and survival.

The developmental requirements for $\mathrm{CD}^{+}$Trm are complex and differ between tissues. In the lung, the formation of Trm cells is TGF $\beta$-dependent ${ }^{3,18,19}$ and requires CD4 ${ }^{+}$T-cell help, $^{20}$ whereas the long-term survival of this memory T-cell population is dependent on IL-15. ${ }^{14}$ Lung Trm develop in distinct niches, associated with tissue remodelling and repair, which occurs after infection-associated lung injury as these regions have elevated levels of expression of Trm-promoting factors, TGF $\beta$ and IL-15. ${ }^{21}$ Local cognate antigen recognition is also required for optimal lung Trm development., ${ }^{3,21-23}$ The local recognition of antigen boosts Trm numbers and also influences the immunodominance hierarchy within the Trm compartment. ${ }^{1,24}$ Whereas in the lung, local antigen recognition is required for Trm development, other

\footnotetext{
${ }^{1}$ Infection and Immunity Program, Monash Biomedicine Discovery Institute and Department of Biochemistry and Molecular Biology, Monash University, Melbourne, VIC, Australia and ${ }^{2}$ Department of Microbiology and Immunology, The University of Melbourne, at the Peter Doherty Institute for Infection and Immunity, Melbourne, VIC 3000, Australia Correspondence: Linda M. Wakim (wakiml@unimelb.edu.au)

These authors contributed equally: Irina Caminschi, Mireille H. Lahoud.
}

Received: 11 May 2018 Revised: 4 November 2018 Accepted: 5 December 2018

Published online: 21 January 2019 
404

tissues, including the skin, ${ }^{25}$ female reproductive tract ${ }^{26}$ and gut, ${ }^{27}$ intrinsically support Trm development and do not require the presence of cognate antigen within the tissue to drive Trm formation.

Several vaccination regimes that promote pulmonary $\mathrm{CD}^{+}$ Trm development have been designed, and these have been shown to effectively limit the severity of respiratory viral infections. ${ }^{1,3,5,21,28-30}$ To date, these approaches all rely on local immunisation with a $\mathrm{CD}^{+}$T-cell-targeted epitope plus adjuvant. Although pulmonary delivery of antigen is important for vaccineinduced lung Trm development, at present, it is unclear if the adjuvant co-administered with the vaccine antigen also impacts Trm development. We set out to investigate how altering the adjuvant co-administered with the pulmonary delivered antigen impacts lung Trm development. Although altering the adjuvant co-administered with the pulmonary delivered antigen did not impact the size of the lung Trm population, strikingly, we found that the intranasal delivery of the adjuvant zymosan, a yeast cell wall derivative, in the absence of antigen, could draw effector $\mathrm{CD}^{+}{ }^{+}$cells into the lung and effectively drive their differentiation into lung Trm. When coupled with an injectable influenza vaccination regime, intranasal zymosan delivery could significantly boost the size of the influenza virus-specific Trm population that persisted within the lung. Overall, these results highlight that the appropriate selection of adjuvant, delivered by the correct route, can greatly improve vaccines that aim to elicit pulmonary Trm.

\section{RESULTS}

Intranasal administration of zymosan drives antigen-independent lung Trm development

Several vaccination strategies that combine the local delivery of antigen plus adjuvant into the airways have been developed and shown to generate highly protective lung Trm. ${ }^{1,3,5,21,29,30}$ Although local cognate antigen recognition is required for the establishment of lung Trm, it is currently unclear how the co-administered adjuvant impacts lung Trm development. To investigate the effect of adjuvant on Trm development, mice were seeded with in vitroactivated ovalbumin (OVA)-specific OT-I CD8 ${ }^{+} \mathrm{T}$ cells, then immunised intranasally with a panel of adjuvants (zymosan, lipopolysaccharide (LPS) and poly $\mathrm{I}: \mathrm{C}$ ) either alone, or in combination with the model antigen OVA. The absolute number of lung Trm, defined by the co-expression of CD69 and CD103 was measured 20 days later (Fig. 1a). Inoculating activated OT-I CD8 ${ }^{+}$ $T$ cells ensured that a large and equivalent number of effector cells, generated in identical conditions, were available for accurate measurement of conversion into Trm cells in all the groups, thus allowing us to exclude the potential influence of each of the adjuvants on $\mathrm{CD}^{+}$T-cell activation. In control mice, which received effector OT-I CD8 ${ }^{+}$T cells without adjuvant or antigen, a baseline of $\sim 1000 \mathrm{CD} 103^{+} \mathrm{CD}^{+} 9^{+}$OT-I Trm cells could be recovered from the lung (Fig. 1a). As expected, the intranasal administration of OVA protein in the presence of adjuvant greatly boosted lung OT-I Trm development, up to 15-20-fold above baseline, irrespective of the co-administered adjuvant (Fig. 1a). Consistent with prior reports that show local cognate antigen recognition is required for efficient lung Trm development, ${ }^{3,21-23}$ intranasal administration of LPS and poly I:C without OVA antigen did not result in a significant increase of lung OT-I Trm above the baseline. However, strikingly, administering zymosan alone could drive lung Trm development to a level comparable to that observed when it was co-administered with the antigen (Fig. 1a). Intravascular staining on these mice revealed that the coexpression of CD69 and CD103 on OT-I cells marked cells in the parenchyma, and that zymosan treatment altered the localisation of memory OT-I cells in the lung, increasing the proportion of parenchyma bound (intravascular antibody negative) memory
OT-I cells from 27\% (saline treated group) to 54\% (zymosan treated group) (Supplementary Figure 1a-d).

We assessed whether zymosan-induced antigen-independent lung Trm development required the adjuvant to be administered into the lung, or whether delivery by alternative routes could also facilitate pulmonary Trm development. To do this, mice seeded with in vitro-activated OT-I CD8 ${ }^{+} T$ cells were administered zymosan either via the intranasal, sublingual or intraperitoneal route, whereas control mice received saline (NIL) intranasally. Twenty days later the number of OT-I T cells that developed into $\mathrm{CD}_{103}{ }^{+} \mathrm{CD}_{69}{ }^{+}$Trm in the lung was enumerated (Fig. 1b). The ability of zymosan to enhance Trm development was dependent on the adjuvant being administered locally into the airways, as delivery by alternative routes failed to boost the number of OT-I Trm above baseline levels observed in the control cohort that received saline (Fig. 1b).

To determine the kinetics of zymosan induced lung Trm development, mice were seeded with in vitro-activated OT-I $T$ cells, then given intranasally zymosan or LPS. In this experimental design, LPS represents an adjuvant that fails to promote antigen-independent Trm development. The number of OT-I T cells in the spleen and lung and their conversion into $\mathrm{CD}_{103}{ }^{+} \mathrm{CD} 9^{+}$Trm was measured at the indicated time points. From days 3 to 6 post transfer, the number of OT-I T cells in the spleen of the LPS- and zymosan-treated mice increased, a likely reflection of the capacity of effector OT-I T cells to undergo several rounds of division following adoptive transfer, however, thereafter, the number of OT-I T cells plateaued and remained stable for the duration of the experiment (Fig. 1c). Although the absolute number of $\mathrm{CD}_{103}{ }^{+} \mathrm{CD} 69^{+}$OT-I Trm in the lung of the LPS treated cohort peaked on day 3 and contracted thereafter, the zymosantreated mice continued to accumulate lung $\mathrm{CD} 103^{+} \mathrm{CD}^{+} 9^{+} \mathrm{Trm}$ OT-I until day 9 post treatment (Fig. 1d-e). In addition to the recruitment of adoptively transferred effector OT-I T cells, zymosan treatment resulted in the recruitment of host cells including endogenous CD8 + and CD4 $+\mathrm{T}$ cells, dendritic cells (DCs), alveolar macrophages, and neutrophils into the lung and airways, however, by day 6 post treatment, the number of these cells were diminishing and were approaching baseline levels (Supplementary Figure 2). We next tracked the longevity of the zymosan-induced OT-I Trm and calculated that these cells have a half-life of 11.5 days, which is comparable to the half-life of lung Trm generated in the presence of antigen following influenza virus infection (12 days ${ }^{1}$ ) (Supplementary Figure 3). All subsets of OT-I memory $T$ cells that persisted in the lung following LPS or zymosan treatment were functionally equivalent, generating similar levels of both tumour necrosis factor (TNF) $a$ and interferon-gamma (IFN- $\gamma$ ) following a brief in vitro peptide stimulation (Fig. $1 \mathrm{f}-\mathrm{g}$ ). Thus, we showed that zymosan, an insoluble glucan purified from yeast cell wall extracts, administered into the airways, can by-pass the requirement for local antigen recognition in the development of lung Trm.

Zymosan signalling through dectin-1 is sufficient to drive antigenindependent lung Trm development

Zymosan signals through a heterodimer of toll like receptor 2 (TLR2) and TLR6 ${ }^{31}$ and the C-type lectin receptor, dectin-1. Although TLR2 is constitutively and widely expressed on many cell types, dectin-1 expression is restricted to myeloid cells. ${ }^{32}$ Consistent with this, we found that in the murine lung, only pulmonary macrophages and $C D 11 b^{+} \mathrm{DCs}$, but not $\mathrm{CD}_{103^{+} \mathrm{DCs}}$ constitutively expressed high levels of the dectin-1 receptor (Fig. 2a-b). We determined which receptor and signalling pathway was required for zymosan-driven antigen-independent lung Trm development. First, we assessed the role of TLR signalling by seeding wild type (WT) or TLR2 knockout mice with in vitroactivated OT-I T cells prior to the intranasal delivery of zymosan. In these experiments, and all future experiments described, we 

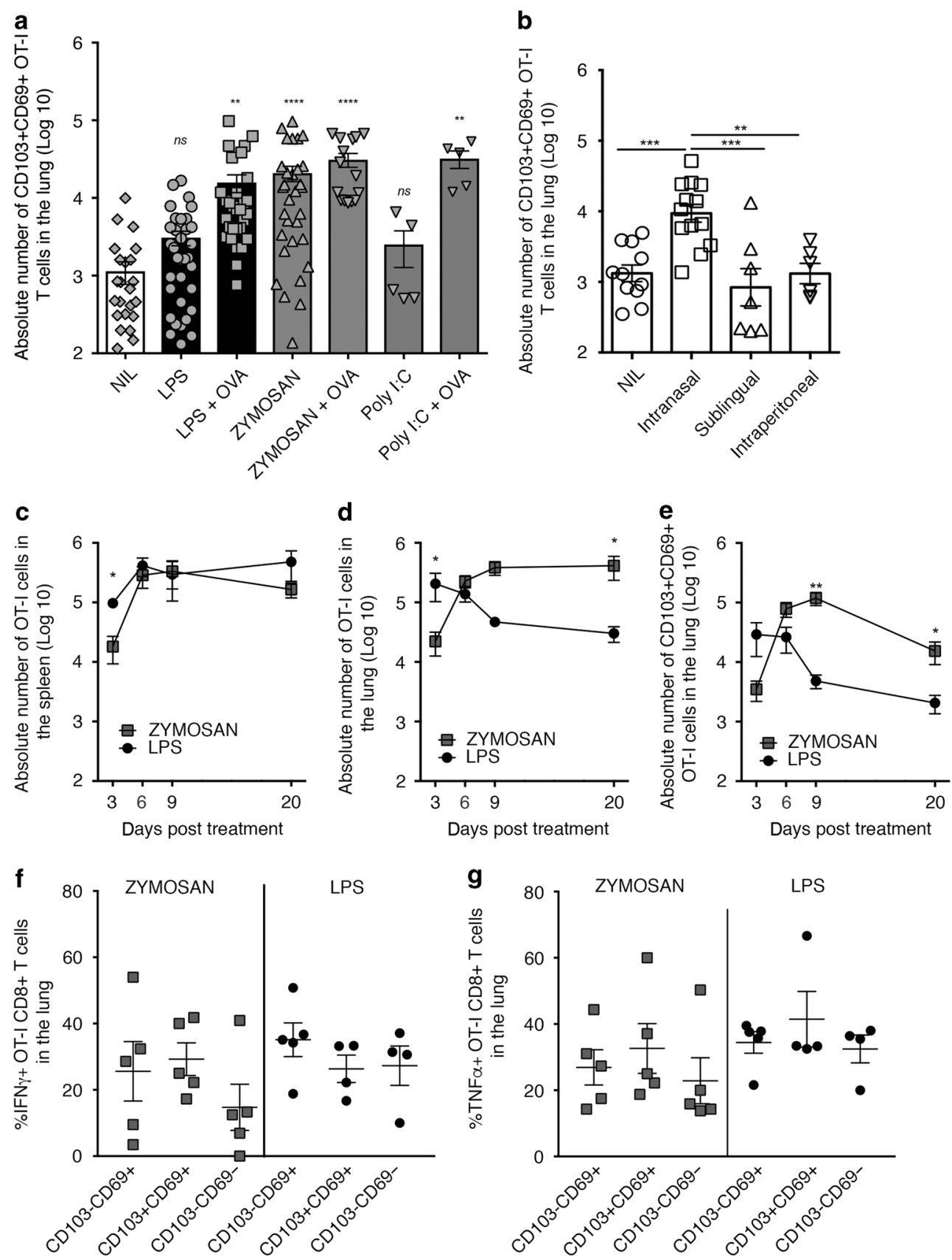

Fig. 1 Intranasal administration of zymosan drives antigen-independent lung Trm development. a Mice seeded with $5 \times 10^{6}$ in vitro-activated OT-I.CD45.1 cells prior to the intranasal administration of either adjuvant (PBS-NIL, LPS, Zymosan or Poly I:C), or adjuvant and OVA protein. On day 20 post treatment, the number of OT-I cells in the lung co-expressing Trm markers (CD69 and CD103) was measured. Data pooled from eight independent experiments with 5-37 mice per group. Bars represent the mean \pm SEM, whereas symbols represent individual mice (oneway ANOVA, Dunnett's multiple comparison). b Mice seeded with $5 \times 10^{6}$ in vitro-activated OT-I.CD45.1 cells prior to the intranasal, sublingual, or intraperitoneal administration of zymosan and on day 20 post treatment the number of OT-I cells in the lung expressing Trm markers (CD69 and $C D 103$ ) was measured. Data pooled from three independent experiments. Bars represent the mean \pm SEM, whereas symbols represent individual mice (one-way ANOVA, Tukey's multiple comparison). c-e Mice seeded with $5 \times 10^{6}$ in vitro-activated OT-I.CD45.1 cells prior to the intranasal administration of zymosan or LPS and at the indicated times the absolute number of OT-I cells in the $\mathbf{c}$ spleen and $\mathbf{d}$ lung and the number of CD103 + CD69 + OT-I Trm cell in the e lung was measured. Data pooled from three independent experiments with 4-10 mice per group (two-way ANOVA, Sidak's multiple comparison). $\mathbf{f}-\mathbf{g}$ OT-I cells isolated from the lungs of mice generated as described in c and harvested 20 days post adjuvant delivery were stimulated in vitro for $4 \mathrm{~h}$ with SIINFEKL peptide and the proportion of $\mathbf{f}$ IFN- $\gamma$ and $\mathbf{g}$ TNF- $\alpha$-producing cells was within each of the memory T-cell subsets differentiated based on expression of CD103 and CD69 was measured. Data pooled from two independent experiments. Bars represent the mean \pm SEM, whereas symbols represent individual mice

delivered a second treatment of zymosan 2 days later as we found it resulted in more consistent Trm conversion. The number of OT-I $\mathrm{T}$ cells in the spleen (Fig. 2c) and lung (Fig. 2d) and their conversion into $\mathrm{CD} 103^{+} \mathrm{CD}^{+} 9^{+}$Trm (Fig. 2e) was measured 30 days later. We observed no significant difference in the number of OT-I $T$ cells and the proportion of conversion of these cells into $\mathrm{CD}_{103}{ }^{+} \mathrm{CD}_{69}{ }^{+}$Trm when comparing the WT and TLR2 KO recipient mice, indicating that zymosan signalling via TLR2 was 


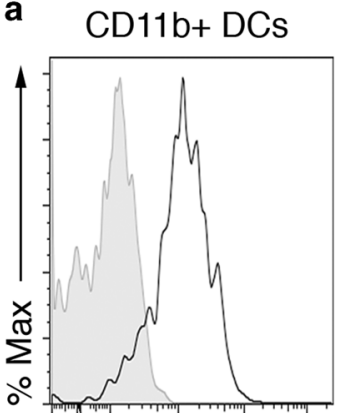

Dectin-1
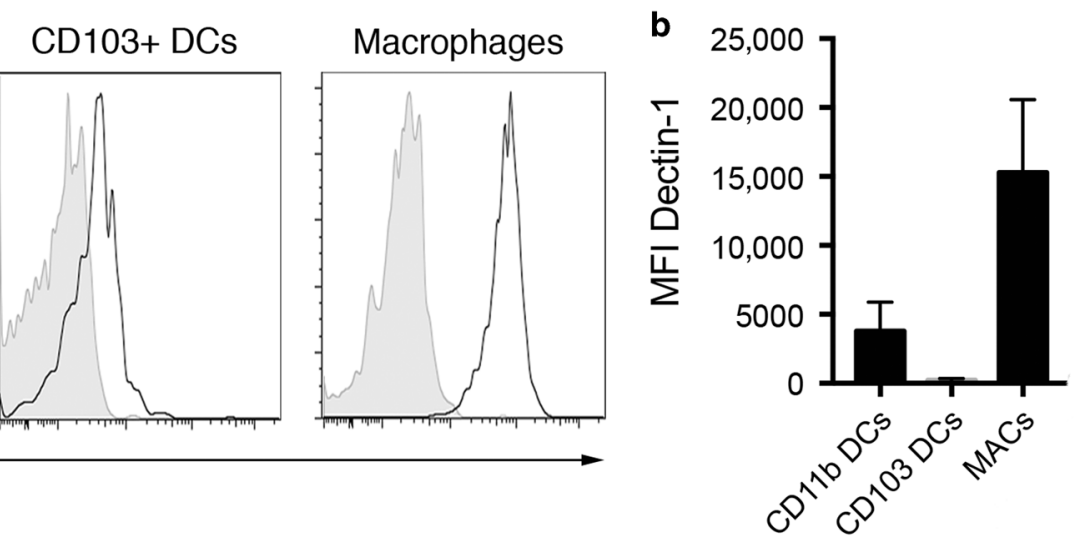

C

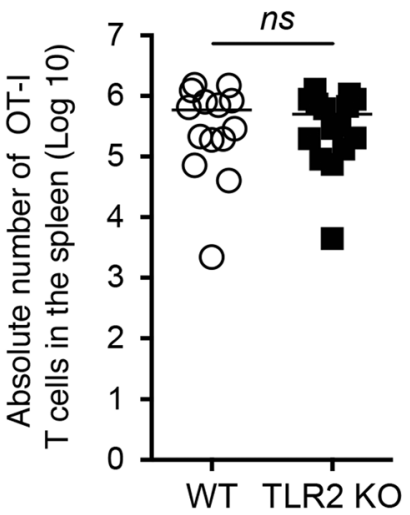

f

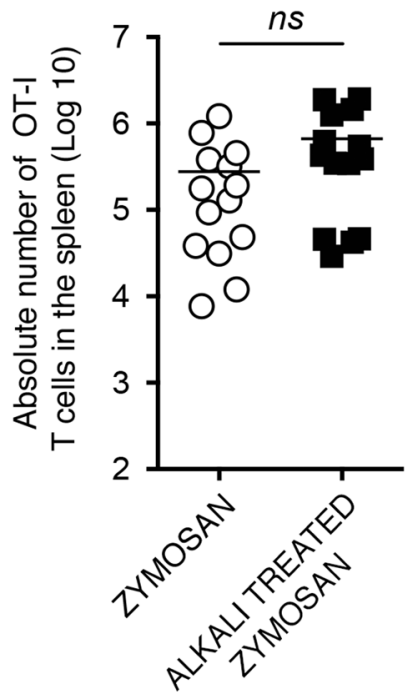

d

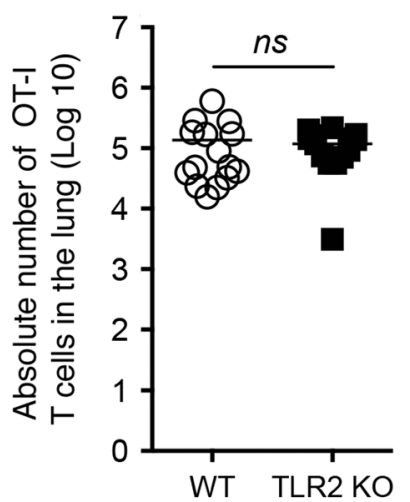

g

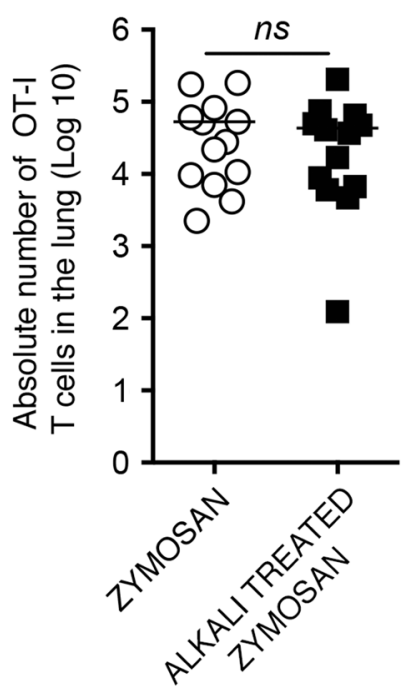

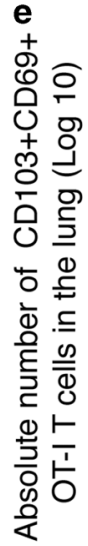

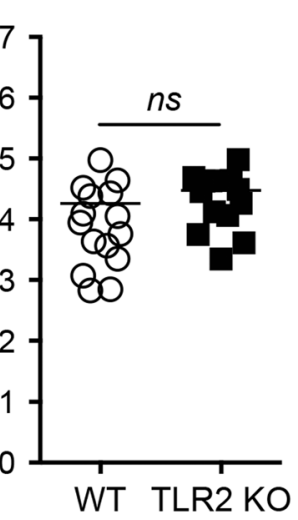

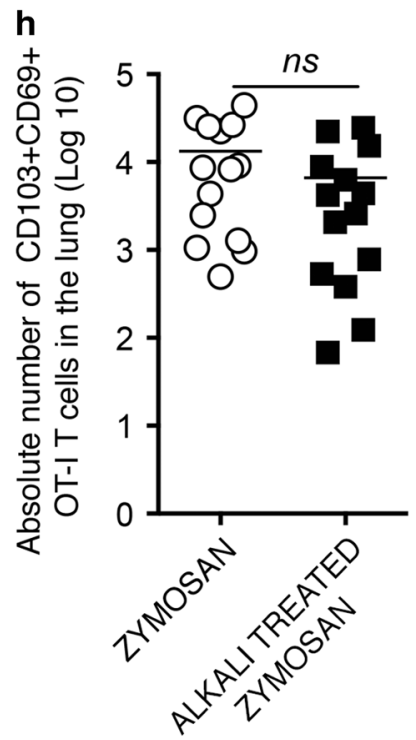

Fig. 2 Zymosan signalling through dectin-1 is sufficient to drive antigen-independent lung Trm development a Representative histograms depict the level of expression of Dectin-1 on CD11b + DCs, CD103 + DCs and macrophages (MHC II + CD64 +) in the lung. Grey histogram shows unstained controls. b MFI of Dectin-1 on dendritic cells and macrophages in the lung. Data pooled from two independent experiments with five mice per group. Bars represent the mean \pm SEM. c-e Mice, either WT or TLR2 KO, seeded with $5 \times 10^{6}$ in vitro-activated OT-I. CD45.1 cells, were administered zymosan i.n. and on day 30 post treatment the total number of OT-I in the $\mathbf{c}$ spleen and $\mathbf{d}$ lung and e the absolute number of CD103 + CD69 + OT-I Trm in the lung was measured. Data are pooled from three independent experiments with 15 mice per group. Symbols represent individual mice with the line representing the mean (student's $t$ test). $\mathbf{f}-\mathbf{h}$ Mice seeded with $5 \times 10^{6}$ in vitroactivated OT-I cells were given either zymosan or alkali treated zymosan i.n. and on day 30 post treatment the total number of OT-I in the $\mathbf{f}$ spleen and $\mathbf{g}$ lung and $\mathbf{h}$ the absolute number of CD103 + CD69+ OT-I Trm in the lung was measured. Data are pooled from three independent experiments with 14 mice per group. Symbols represent individual mice with the line representing the mean (student's $t$ test) 
not required for the antigen-independent Trm development (Fig. 2e). To further exclude the requirement of TLR signalling in zymosan induced lung Trm development, we seeded wild type (WT) mice or mice that lacked Myd88, an adaptor protein that is critical for TLR receptor family signalling, with in vitro-activated OT-I T cells prior to the intranasal delivery of zymosan. Quantitation of the number of OT-I T cells in the spleen (Supplementary Fig 4) and lung (Supplementary Fig 4) and their conversion into $\mathrm{CD}_{103}{ }^{+} \mathrm{CD} 9^{+}$Trm (Supplementary Fig 4) measured 30 days later revealed no significant difference in the number of OT-I T cells and the proportion of conversion of these cells into $\mathrm{CD}_{103}{ }^{+} \mathrm{CD}_{69}{ }^{+}$Trm when comparing the WT and Myd88 $\mathrm{KO}$ recipient mice, further indicating that TLR signalling was not required for zymosan induced antigen-independent Trm development (Supplementary Fig 4)

Next, to assess if zymosan signalling via dectin-1 alone was sufficient to drive lung Trm development, WT mice seeded with in vitro-activated effector OT-I T cells were treated intranasally with either standard zymosan, or alkali-treated zymosan, which lacks TLR2 stimulating properties while preserving dectin-1 activity. As above, the number of OT-I T cells in the spleen (Fig. 2f) and lung (Fig. $2 \mathrm{~g}$ ) and their conversion into lung $\mathrm{CD}_{103}{ }^{+} \mathrm{CD} 69^{+} \mathrm{Trm}$ (Fig. 2h) was measured 30 days later. Animals administered standard zymosan or treated zymosan had similar numbers of memory OT-I T cells in their spleen and lung and equivalent numbers of lung $\mathrm{CD}_{103}{ }^{+} \mathrm{CD} 69^{+} \mathrm{OT}-\mathrm{I}$ Trm. Although zymosan signalling via dectin-1 was sufficient for antigen-independent lung Trm development, further experiments using the dectin-1deficient mice are required to formally demonstrate if this signalling pathway has an essential and non-redundant role in this process.

Zymosan induces local inflammation, the recruitment of neutrophils into the lung and drives antigen-independent but TGF $\beta$-dependent lung Trm development

A temporal assessment of the level of a panel of inflammatory chemokines and cytokines (Interleukin (IL)-6 (IL-6), IL-10, IL-12p70, monocyte chemoattractant protein-1 (MCP-1), IFN- $\gamma$ and TNF) within the airways of mice intranasally administered either zymosan or, as a control LPS, revealed IL-6, MCP-1, and TNF were significantly elevated in bronchial alveolar lavage of zymosan treated animals between 24 and $48 \mathrm{~h}$ post adjuvant delivery (Fig. 3a), reflecting the capacity of zymosan to induce robust pulmonary inflammation. ${ }^{33}$ The other cytokines profiled were not increased by either stimulation. To assess the immune cells infiltrating the lung following intranasal zymosan delivery, mice were intranasally administered either zymosan or, as a control LPS, and at the indicated time points the number of monocytes

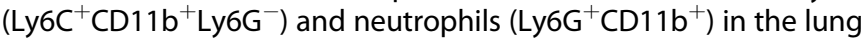
were enumerated. We observed no significant difference in the infiltration and persistence of monocytes in the lungs of animals administered either LPS or zymosan (Fig. 3b). However, although there were equivalent numbers of neutrophils infiltrating the lung of zymosan and LPS-treated animals for the first $48 \mathrm{~h}$ post treatment, the number of neutrophils in mice administered LPS declined thereafter, whereas neutrophils numbers continued to increase in the zymosan-treated mice remaining elevated for at least 6 days post zymosan delivery (Fig. $3 \mathrm{C}$ ). To investigate the role of neutrophils in zymosan induced antigen-independent lung Trm development, control mice or mice depleted of neutrophils, were seeded with in vitro-activated OT-I T cells prior to the intranasal delivery of zymosan, and on day 20 post treatment the number of OT-I T cells that had differentiated into lung $\mathrm{CD} 103^{+} \mathrm{CD} 69^{+} \mathrm{Trm}$ was measured. As there was no difference in the number of OT-I $T$ cells that converted into lung Trm in the animals that were depleted of neutrophils compared with the control cohort we could exclude a direct involvement of neutrophils in zymosan driven antigen-independent lung Trm development (Fig. 3d).
To gain further insight into the cells within the lung responding to zymosan, we intranasally administered zymosan particles that were covalently conjugated to the fluorophore alexa594 and over time, determined which cells engulfed these fluorescent particles. Immunofluoresence microscopy on the lung tissue 2 days following intranasal delivery of zymosan particles showed extensive cellular infiltration surrounding patches of fluorescently tagged zymosan particles (Fig. 3e). Flow cytometric assessment of lung homogenates 2 days post administration showed that the majority of fluorescent zymosan particles were equally distributed among Ly6G + neutrophils and CD64 + macrophages, although 6-9 days post administration, the fluorescent zymosan particles were solely associated with pulmonary macrophages (Fig. 3f).

Previous reports show that zymosan induces $\mathrm{F} 4 / 80^{+}$macrophages in the splenic red pulp to secrete TGF $\beta$ - a cytokine known to promote Trm development. ${ }^{34}$ We assessed if pulmonary macrophages secreted TGF $\beta$ following zymosan delivery. To do this, we intranasally administered fluorescent zymosan particles (alexa594) and 2 days later we compared the expression of the latency-associated peptide (LAP) on CD64 + macrophages that had engulfed zymosan particles (alexa594 +) to those isolated from the same lung tissue that had not engulfed zymosan (alexa594-). TGF $\beta$ is secreted in its latent form as a homodimer non-covalently associated with the LAP. Surface expression of LAP on other immune cells has been used successfully as an indirect assessment of surface bound TGF $\beta .{ }^{35}$ The vast majority ( $>93 \%$ ) of LAP + cells present in the lung following zymosan treatment were alveolar macrophages (Supplementary Fig 5). We observed that the mean fluorescent intensity of LAP was higher on CD64+ macrophages that had engulfed zymosan particles compared with macrophages that had not engulfed zymosan particles, which was indicative of TGF $\beta$ synthesis by pulmonary macrophages following zymosan exposure (Fig. $3 g-h$ ).

To further investigate the involvement of TGF $\beta$ signalling in zymosan-induced lung Trm development, we seeded mice with either in vitro-activated WT OT-I T cells or activated OT-I T cells that were deficient in expression of the TGF $\beta$ receptor (TGF $\beta$ RII $\mathrm{KO})$, just prior to intranasal delivery of either zymosan or as a control saline. On day 30 post treatment, the number of OT-I T cells that had converted into $\mathrm{CD} 103^{+} \mathrm{CD} 69^{+}$Trm was measured. Consistent with our earlier experiments (Fig. 1a), a baseline of $\sim 1000 \mathrm{CD} 103^{+} \mathrm{CD}^{+} 9^{+}$WT or TGF $\beta$ RII KO OT-I Trm cells could be recovered from the lung of animals that did not receive any adjuvant. Although the intranasal delivery of zymosan boosted the

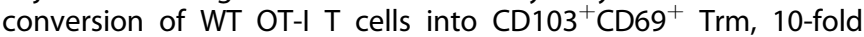
above levels observed in animals that did not receive any adjuvant, zymosan could not drive the conversion of TGF $\beta$ receptor deficient OT-I cells into $\mathrm{CD}_{103}{ }^{+} \mathrm{CD} 69^{+}$Trm (Fig. 3i). Thus, zymosan-induced antigen-independent lung Trm development was dependent on TGF $\beta$.

\section{Zymosan treatment during influenza virus infection boosts Trm} development

We next addressed whether zymosan could boost the number of lung Trm if administered during a respiratory virus infection. To do this, mice were infected via the intranasal route with $10^{4}$ plaque forming units (PFU) of influenza virus (X31, H3N2 strain) and on days 7 and 10 post infection either zymosan, LPS or saline was administered into the airways and at day 30 post infection the number of influenza-specific $\mathrm{CD} 8^{+} \mathrm{T}$ cells that developed into Trm were enumerated. We tracked the influenza virus-specific $\mathrm{CD}^{+}$ T-cell response against two immunodominant epitopes derived from the viral nucleoprotein $\left(\mathrm{NP}_{366}\right)$ and acid polymerase $\left(\mathrm{PA}_{224}\right)$ using $\mathrm{H}-2 \mathrm{D}^{\mathrm{b}}$ tetramers loaded with the $\mathrm{PA}_{224}$ and $\mathrm{NP}_{366}$ epitopes. We have recently reported that $\mathrm{PA}_{224^{-}}$and $\mathrm{NP}_{366^{-}}$specific $\mathrm{CD} 8^{+}$ $\mathrm{T}$ cells differentiated into Trm with varying efficiencies ${ }^{1}$ and consistent with those findings, here we show that although $30 \%$ of PA-tetramer ${ }^{+} \mathrm{T}$ cells differentiate into Trm only $\sim 6 \%$ of the 
I Caminschi et al.

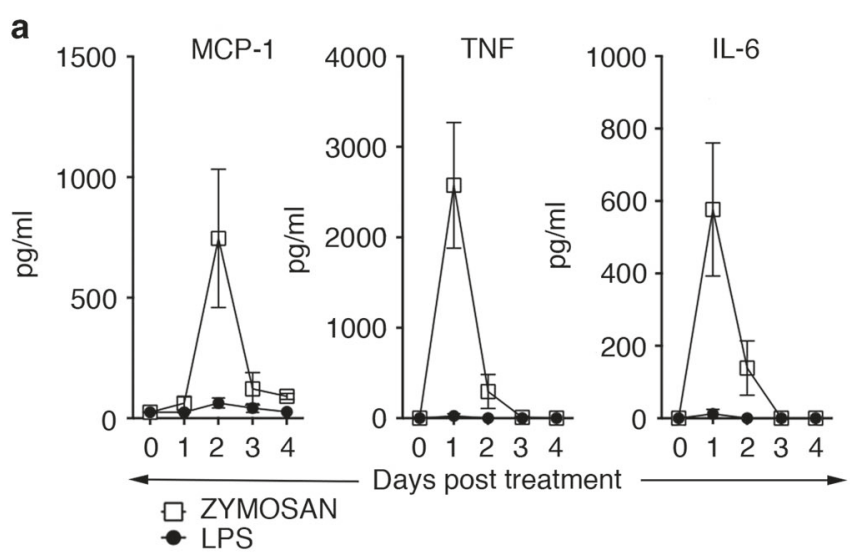

C

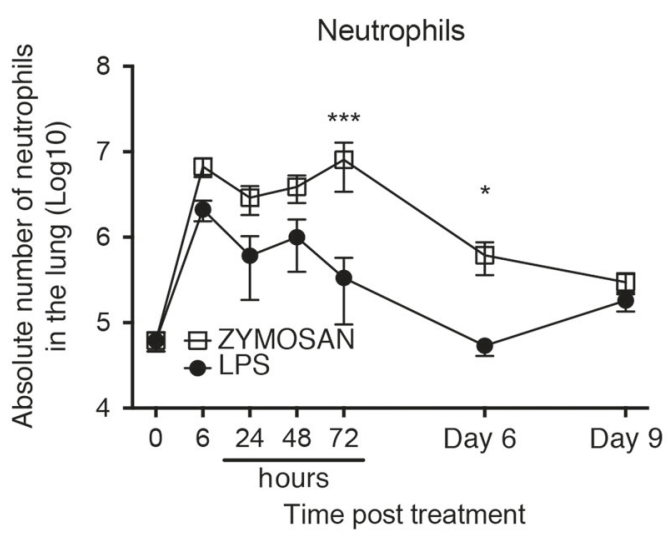

$\mathbf{f}$

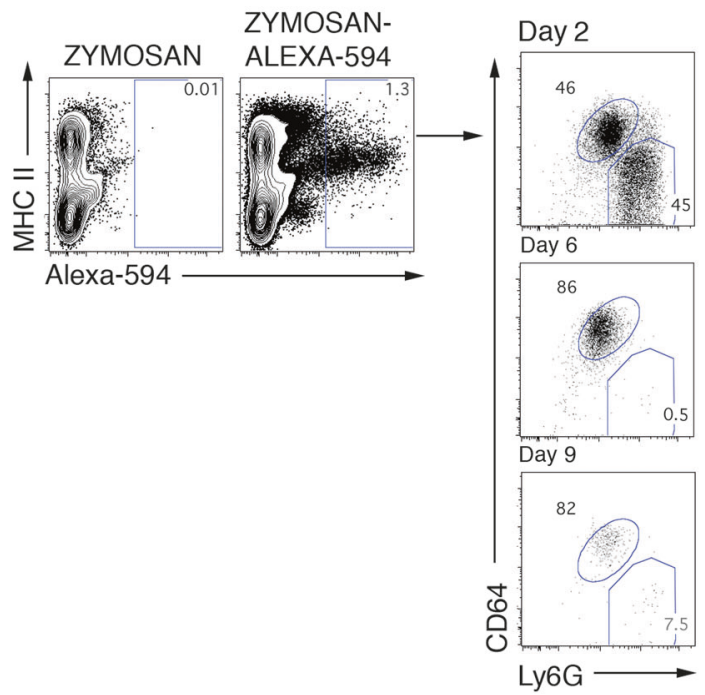

g

h

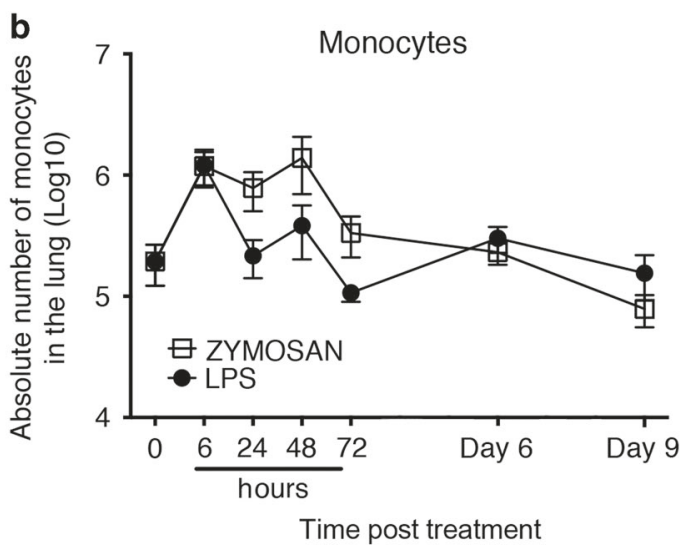

e
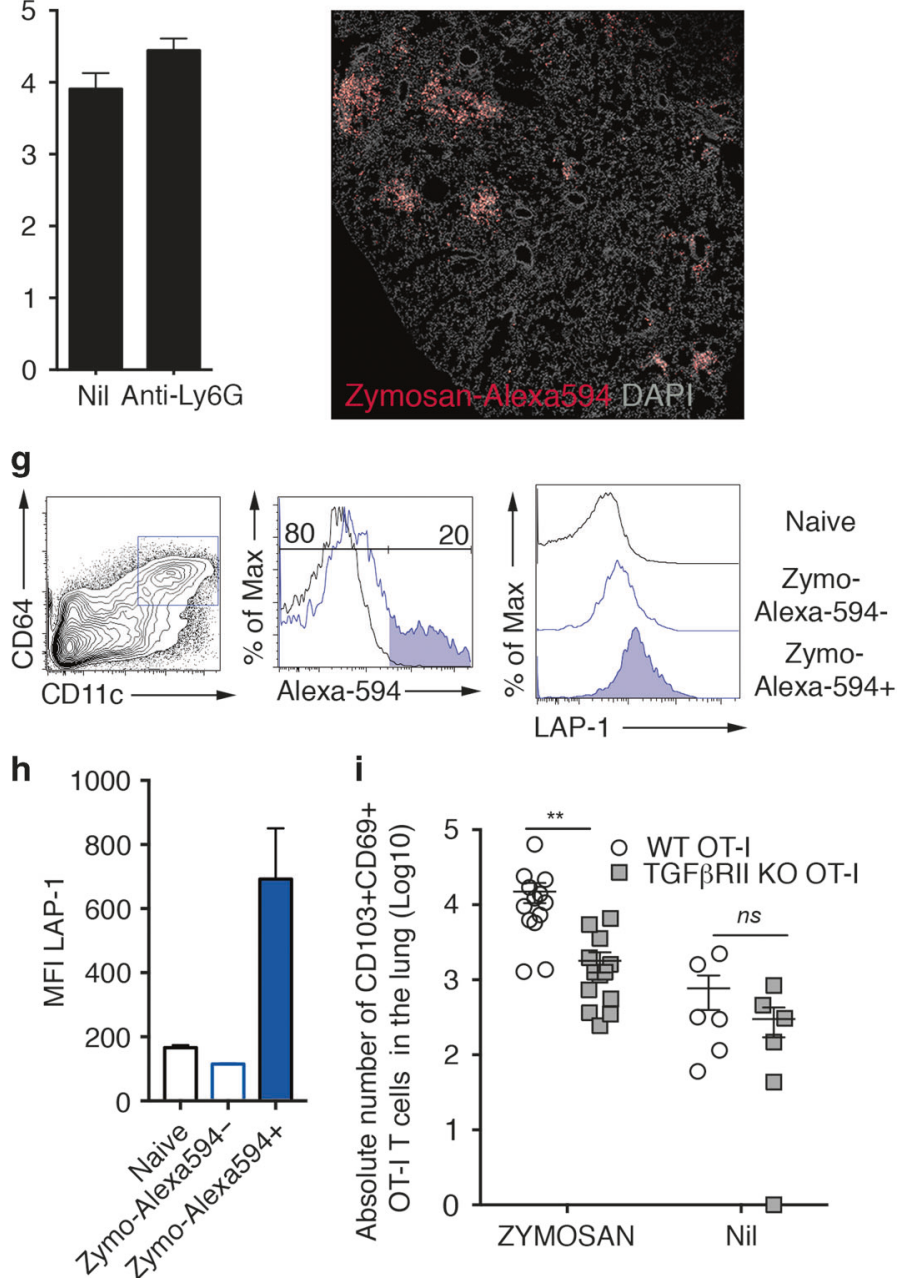

i

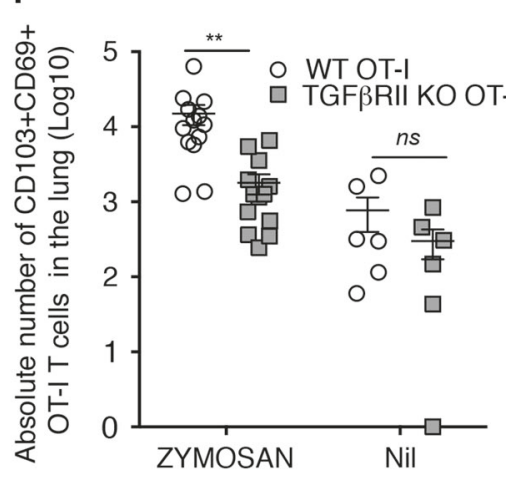

NP-specific T cells adopted a Trm phenotype (Fig. 4a). Whereas administering zymosan intransally during the acute phase of influenza virus infection did not impact the size of the NP- or PAtetramer ${ }^{+}$T-cell pool in the spleen (Fig. 4b), it did boost the total number NP-tetramer ${ }^{+} \mathrm{T}$ cells in the lung and significantly increased the proportion that developed into Trm (Fig. 4a, C, d). We next assessed the $\mathrm{V} \beta$-usage of the $\mathrm{PA}_{224}$ and $\mathrm{NP}_{366}$-specific memory CD8 $T$ cells to determine whether zymosan treatment introduced a bias in the Trm repertoire. We found the $\mathrm{PA}_{224}$ and $\mathrm{NP}_{366}$-specific memory $\mathrm{CD} 8+\mathrm{T}$ cells in the lung generated in the presence or absence of zymosan displayed a similar pattern of $V \beta$ usage (Supplementary Fig 6), which suggests that zymosan treatment does not alter the T-cell repertoire but simple boosts the number of cells transitioning into Trm.

We next assessed if intranasal zymosan treatment could drive influenza-specific lung Trm development, when coupled with an injectable influenza vaccination regime. To do this, mice were first primed with influenza virus by injecting $10^{7} \mathrm{PFU}$ of influenza virus (PR8, H1N1 strain) into the peritoneal cavity. Injection via this route is non-productive, and while it results in effective priming of 
Fig. 3 Zymosan induces local inflammation, the recruitment of neutrophils into the lung and drives antigen-independent but TGF $\beta$ dependent Trm development. a Bronchial alveolar lavage fluid from mice administered zymosan or LPS i.n. was harvested on days 0-4 post treatment and the level of inflammatory cytokines was measured using a cytometric bead array. Data pooled from three independent experiments with 4-11 mice per group. Symbols represent the mean \pm SEM. b-c Mice were given zymosan or LPS intranasally and at the indicated time points the absolute number of $\mathbf{b}$ monocytes (Ly6c + Ly6g-CD11b + ) and $\mathbf{c}$ neutrophils (Ly6G + CD11b + ) in the lung was measured. Data pooled from three independent experiments with 4-7 mice per group (two-way ANOVA, Sidak's multiple comparison). $\mathbf{d}$ Mice seeded with $5 \times 10^{6}$ in vitro-activated OT-I.CD45.1 cells prior to the intranasal administration of zymosan were injected with anti-Ly6G (1A8) (neutrophil depletion antibody) or given PBS (NIL) every second day for the duration of the experiment. On day 20 post treatment the number of OT-I cells in the lung expressing Trm markers (CD69 and CD103) was measured. Data pooled from two independent experiments. Bars represent the mean + SEM with six mice per group. e I.H.C of frozen lung sections from mice given zymosan-alexa594 and harvested on day 2 post treatment (zymosan-alexa594, and DAPI). f Mice were intranasally instilled with zymosan-alexa594 and on days 2-9 post treatment the lungs were harvested, digested and alexa594+ cells were profiled using flow cytometry and identified as predominately CD64+ macrophages and Ly6G + neutrophils. g-h Mice received zymosan-alexa594 intranasally and 2 days post treatment the level of LAP-1 expression on CD64 + CD11c + alveolar macrophages that had engulfed zymosan was measured. $\mathbf{g}$ Representative histograms demonstrated the level of expression of LAP-1 on alveolar macrophages that had engulfed zymosam (zymo-alexa594 +) compared with macrophages recovered from the same lung tissue that had not engulfed zymosan (zymo-alexa594-) or macrophages recovered from the lungs of naive mice. $\mathbf{h}$ Data pooled from two experiments with four mice per group showing the MFI of LAP-1 in zymosan-alexa594 + and zymosanalexa594- macrophages. i Mice were seeded with $5 \times 10^{6}$ in vitro-activated WT or TGF $\beta$ RII KO OT-I.CD45.1 cells and administered intranasally zymosan or PBS (NIL). On day 30 post treatment the total number of CD103 + CD69 + OT-I Trm in the lung was measured. Data are pooled from three independent experiments with 6-14 mice per group. Symbols represent individual mice, lines represent mean \pm SEM (two-way ANOVA, Sidak's multiple comparison)

influenza-specific $\mathrm{CD}^{+} \mathrm{T}$ cells, it does not lodge influenza-specific Trm within the lung., ${ }^{5,2}$ On day 7 and 10, p.i mice were administered zymosan intranasally, or as controls either LPS or saline (NIL), and the number of influenza-specific NP-tetramer ${ }^{+}$ $\mathrm{CD}^{+} \mathrm{T}$ cells in the spleen and lung and the proportion that differentiated into lung $\mathrm{CD}_{103^{+}} \mathrm{CD} 69^{+}$Trm was measured 30 days later. In comparison with the control groups that were administered no adjuvant or LPS, the zymosan treatment group showed reduced numbers of NP-tetramer ${ }^{+} \mathrm{T}$ cells in the spleen (Fig. 4e). Although there was no statistically significant difference in the numbers of total NP-tetramer ${ }^{+} \mathrm{T}$ cells in the lung between the three cohorts (Fig. 4f), assessment of the proportion that developed into $\mathrm{CD}_{103}{ }^{+} \mathrm{CD} 69^{+} \mathrm{Trm}$ revealed that zymosan treatment boosted Trm development 25-fold above levels observed following administration of LPS (Fig. 4g). Thus, when combined with an injectable influenza vaccination regime, intranasal zymosan delivery can significantly boost the size of the influenza virus-specific lung Trm population.

\section{DISCUSSION}

Vaccination strategies that deposit Trm along the airways are likely to confer protective immunity against respiratory pathogens. A critical step towards the development of such a vaccine is the identification of factors that promote pulmonary Trm formation. Although local cognate antigen recognition has been shown to be important in driving lung Trm development, ${ }^{3,21-23}$ currently it is not clear if other local cues also guide the formation of pulmonary Trm. Here, we investigated whether the inflammatory milieu in the lung influences the Trm pool. To do this, we evoked different inflammatory profiles within the lung by changing the adjuvant co-administered with an intranasal vaccine and investigated how this affected lung Trm development. While altering the adjuvant co-administered with the pulmonary delivered vaccine did not impact the size of the lung Trm population, a likely reflection that local antigen recognition drives the deposition of the maximum number of Trm, here we identified an adjuvant, zymosan, that when administered into the airways alone could effectively drive the differentiation of immigrating effector $\mathrm{CD}^{+} \mathrm{T}$ cells into lung Trm, thereby, overriding the requirement for local cognate antigen recognition in lung Trm development.

Zymosan is an insoluble fungal cell wall extract that has been shown to have anti-inflammatory properties. The injection of zymosan in vivo induces regulatory antigen-presenting cells, which display a marked reduction in their phagocytic activity, ${ }^{36}$ reduced levels of secretion of pro-inflammatory molecules and elevated levels of expression of suppressive cytokines, including IL-10 and TGF $\beta^{37-40}$ - these cells promote immune tolerance. ${ }^{34,40}$ The anti-inflammatory effects of zymosan make it an interesting candidate for the treatment of autoimmune diseases. In a murine model of multiple sclerosis, zymosan treatment alone suppressed MHC class II upregulation, inhibited upregulation of co-stimulatory molecules on dendritic cells, and promoted differentiation of regulatory $\mathrm{T}$ cells (Tregs), which were biologically active in inhibiting encephalitogenic T-cell mediated autoimmune disease. $^{41}$ Moreover, a single injection of zymosan just prior to diabetes onset significantly delayed the progression of disease in NOD mice; this protection was attributed to the ability of zymosan to evoke TGF $\beta$ and promote the generation of Foxp3 + Tregs. $^{35}$ In contrast to studies that deliver zymosan during T-cell priming, the outcome of which induced impaired T-cell responses and promoted tolerance, here we delayed delivery of zymosan until after T-cell priming had occurred or introduced zymosan with preactivated effector $\mathrm{CD}^{+} \mathrm{T}$ cells. In this context, zymosan promoted the differentiation of effector $T$ cells into long lived Trm. In general, during a localised infection, Trm develop within tissue environments where there are large amounts of regulatory cytokines being produced, which serve to dampen the immune response following infection resolution in order to prevent excessive tissue damage. Thus, anti-inflammatory environments are likely to support Trm development. Strategies that can switch the local tissue environment from pro- to anti-inflammatory, may effectively divert immigrating T cells away from becoming shortlived effectors and promote their conversion into long lived Trm.

Within various tissues, including the gut, ${ }^{27}$ skin, $^{25}$ nasal tissue ${ }^{1}$ and the female reproductive tract, ${ }^{26}$ effector $\mathrm{CD}^{+}{ }^{+}$T cells develop into Trm irrespective of local cognate antigen recognition. In others, including the lung ${ }^{3,21-23}$ and brain, ${ }^{42}$ local antigen recognition is essential for the formation of Trm, affecting both abundance and composition by fine tuning the selection of cells into the Trm compartment and driving the differentiation of cells best suited to recognise antigens presented in the tissue. ${ }^{1,24}$ It is currently unclear why certain tissues require local antigen recognition for Trm development whereas others intrinsically support Trm lodgement and survival. Furthermore, it was not known whether altering the inflammatory profile in a tissue environment could override this requirement for local antigen stimulation in Trm development. Here, we show that introducing zymosan into the lung, a tissue previously classified as an environment that supports antigen-dependent Trm development, 

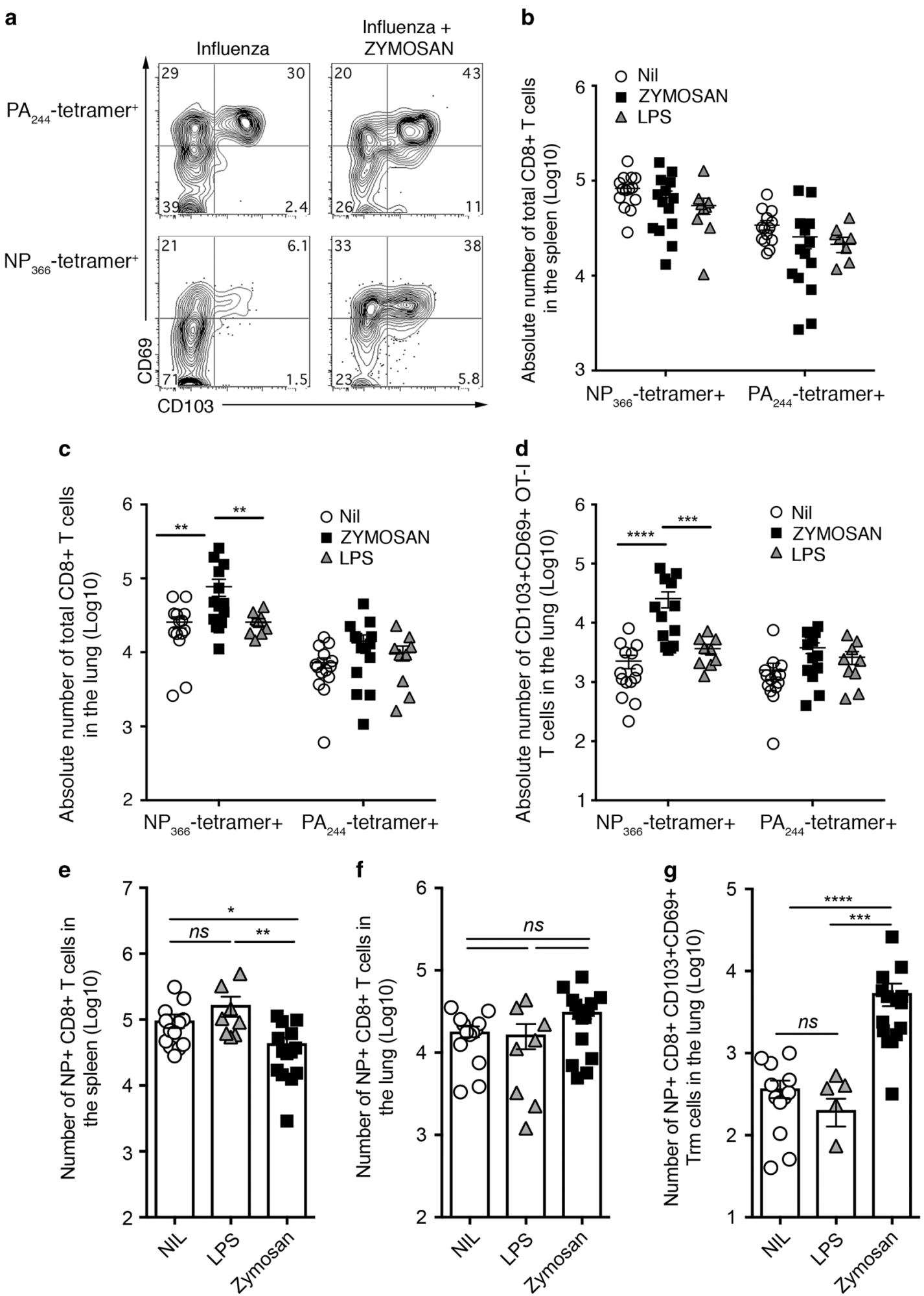

Fig. 4 Zymosan treatment during influenza virus infection boosts Trm development. Mice, infected intranasally with $10^{4}$ PFU X31 influenza virus were administered either, LPS or zymosan or PBS (Nil) on days 7 and 10 post infection and on day 30 post infection the lung and spleen were harvested. a Representative flow cytometry profiles profiling CD103 and CD69 expression on $\mathrm{PA}_{244}$-tetreamer + and $\mathrm{NP}_{366}$-tetramer + $\mathrm{CD} 8+\mathrm{T}$ cells in the lung. The absolute number of $\mathrm{PA}_{244}$-tetreamer + and $\mathrm{NP}_{366}$-tetramer $+\mathrm{CD} 8+\mathrm{T}$ cells in the $\mathbf{b}$ spleen and $\mathbf{c}$ lung and the absolute number of CD103 + CD69 + Trm PA 244 -tetreamer + and $\mathrm{NP}_{366}$-tetramer $+\mathrm{CD} 8+\mathrm{T}$ cells in the d lung was measured. Data pooled from three independent experiments with 9-14 mice per group. Symbols represent individual mice, lines represent mean \pm SEM (two-way ANOVA, Sidak's multiple comparison). e-g Mice, infected via the intraperitoneal route with $10^{7}$ PFU PR8 influenza virus and on day 7 and 10 post infection were administered either, LPS, zymosan or PBS (Nil) and on day 30 post infection the absolute number of NP-tetramer + CD8 T cells in the e spleen and f lung and $\mathbf{g}$ NP-tetramer + CD103 + CD69+Trm in the lung was measured. Data pooled from three independent experiments with 8-13 mice per group. Symbols represent individual mice, bars represent mean \pm SEM (one-way ANOVA, Tukey's multiple comparison) 
can by-pass this antigen dependency and alone drive effector $\mathrm{CD}^{+}{ }^{+} \mathrm{T}$-cell differentiation into Trm. The intranasal delivery of zymosan induces TGF $\beta,{ }^{34,35}$ a cytokine known to be important in the differentiation of Trm, and this cytokine was critical for antigen-independent lung Trm development. In addition, the delivery of zymosan into the airways is known to induce acute lung injury. ${ }^{43}$ Recent studies report that $\mathrm{CD}^{+}$Trm in the lung develop within specific niches associated with tissue repair and remodelling, which occurs following infection. ${ }^{21}$ The damage induced by this adjuvant and the corresponding "regeneration profile" associated with tissue injury repair is likely to further support lung Trm development. Thus, the capacity of zymosan to promote lung Trm likely stems from the ability of this adjuvant to induce both tissue damage and evoke an anti-inflammatory environment.

Here we show that the appropriate selection of adjuvant, delivered by the correct route, can greatly improve vaccines that aim to elicit pulmonary Trm. Zymosan itself is unlikely to represent an adjuvant that transitions easily into the clinic-it is insoluble and higher doses are known to cause acute lung injury. Furthermore, the lung is a fragile environment, excessive inflammation elicited by the pulmonary administration of vaccine adjuvants like zymosan may result in robust cellular infiltration which could damage the lung tissue-a situation that could have a negative impact on respiratory health. However, greater understanding of how zymosan promotes Trm development will allow for the generation of refined adjuvants that evoke conditions, which favour Trm development with minimal side effects.

\section{METHODS}

Mice and viruses

C57BL/6 (CD45.2), Myd88 KO, TLR2 KO (a kind gift from D. Jackson, University of Melbourne, Australia), Tgfbr2 ${ }^{\mathrm{f} / \mathrm{f}} \mathrm{dLck}$-Cre.OT-I.CD45.1 and OT-I.CD45.1, mice were bred in-house and housed in specific pathogen-free conditions in the animal facility at the Doherty Institute of Infection and Immunity, the University of Melbourne. All experiments were done in accordance with the Institutional Animal Care and Use Committee guidelines of the University of Melbourne. Mice were infected intranasally with X31 (generously provided by Dr. S. Turner, Monash University, Melbourne, Australia). Mice were anaesthetized with inhalation anaesthetic and infected with $10^{4}$ PFU of X31 in a volume of $30 \mu \mathrm{l}$. For intraperitoneal infection, mice were infected with $10^{7}$ PFU of PR8 (generously provided by Dr. S. Turner, Monash University, Melbourne, Australia).

In vitro activation of OT-I

A total of $5 \times 10^{7}$ transgenic splenocytes from OT-I mice were cultured for 4 days with $5 \times 10^{7} \mathrm{C} 57 \mathrm{BL} / 6$ splenocytes pulsed with $10 \mu \mathrm{g}$ of OVA $257-264$ peptide, in $40 \mathrm{ml}$ of RPMI 1640 supplemented with $10 \%$ fetal calf serum (FCS), $2 \mathrm{~mm}$ glutamine, $5 \times 10^{-5} \mathrm{M} 2-\mathrm{ME}$ and antibiotics. The cultures were diluted 1 in 2 on days 2 and 3 with fresh medium containing $10 \mathrm{U} / \mathrm{ml} \mathrm{IL-2}$. On day 4 , cells were collected, and $5 \times 10^{6} \mathrm{~T}$ cells were transferred into recipient mice via i.v. injections in a total volume of $200 \mu$ l of phosphate-buffered saline (PBS) just prior to adjuvant and antigen delivery.

\section{Adjuvants and immunisations}

Mice were administered intranasally in volume of $50 \mu \mathrm{l}, 300 \mu \mathrm{g}$ of OVA (Worthington) in combination with the following doses of adjuvants: $100 \mu \mathrm{g}$ zymosan A (Saccharomyces cerevisiae, life technologies), $100 \mu \mathrm{g}$ Zymosan A- alexa fluor-594 (S. cerevisiae, life technologies), $100 \mu \mathrm{g}$ Hot alkali treated zymosan (InvivoGen), $1 \mu \mathrm{g}$ of LPS (Escherichia coli, Sigma), $20 \mu \mathrm{g}$ poly I:C (InvivoGen). To deplete neutrophils mice were injected i.p. with $0.5 \mathrm{mg}$ of antiLy6G (1A8) every second day for the duration of the experiment.
Immunohistochemistry

Perfused lung tissue, inflated with optimal cutting temperature (OCT) were immediately embedded in OCT. Frozen sections (14 $\mu \mathrm{m})$ were cut using a cryostat. Tissue sections were acetone fixed, and blocked in serum-free protein block.

\section{Flow cytometry and cell sorting}

Single-cell suspensions were prepared from spleens by mechanical disruption. Mice were perfused prior to the harvest of the lung tissue, which were enzymatically digested for $1 \mathrm{~h}$ at $37{ }^{\circ} \mathrm{C}$ in $3 \mathrm{ml}$ of collagenase type 3 (Worthington), ( $3 \mathrm{mg} / \mathrm{mL}$ in RPMI 1640 supplemented with $2 \%$ FCS) and DNAse I $(0.5 \mathrm{mg} / \mathrm{ml})$ (Roche). Cells were stained for $25 \mathrm{~min}$ on ice with the appropriate mixture of monoclonal antibodies and washed with PBS with $1 \%$ bovine serum albumin. The conjugated monoclonal antibodies were obtained from BD Pharmingen, eBioscience or Miltenyi include; anti-CD8 (53-6.7), anti-CD8 (YTS-169.4), anti-CD45-1 (A20), anti-CD103 (2E7), anti-Ly6G (1A8), anti-CD11b (M1/70), anti-CD69 (H1.2F3), anti-CD64 (X54-5/7.1), anti-I-A/I-E (M5/114), anti-dectin-1 (REA154).

$\mathrm{H} 2-\mathrm{D}^{\mathrm{b}}$-NP or $\mathrm{H}_{2}-\mathrm{D}^{\mathrm{b}}-\mathrm{PA}$ tetramers were made in house. The levels of IFN- $\gamma$, TNF- $a$, IL-6, IL-10, IL-12p70 and MCP-1 in bronchoalveolar lavage fluid were analysed using a BD Cytometric Bead Array Mouse Inflammation Kit (BD Biosciences, San Diego, (A, USA) according to the manufacturer's instructions. V $\beta$-usage analysis was performed using a BD Mouse Vb TCR Screening Panel Kit (BD Biosciences, San Diego, CA, USA) according to the manufacturer's instructions. Samples were acquired using a Becton Dickinson Fortessa III flow cytometer and data were analysed using the FlowJo software package (Tree Star, Inc., Ashland, OR, USA).

Anti-CD8 antibody in vivo labelling

Mice were injected intravenously with $3 \mu \mathrm{g}$ of phycoerythrinconjugated antibody to CD8 (clone YTS-169) 5 min before they were killed. Mice were perfused with PBS, and tissues were collected, processed and stained with allophycocyanin-conjugated antibody to CD8 (anti-CD8; clone 53-6.7, eBioscience).

Statistical analysis

Data were analysed with GraphPad Prism and the indicated statistical tests. ${ }^{*} P<0.05,{ }^{* *} P<0.01,{ }^{* * *} P<0.001$.

\section{ACKNOWLEDGEMENTS}

We thank Dr. D. Jackson (Melbourne University, Australia) for TLR2 KO mice and Dr. S Turner (Monash University, Australia) for influenza viruses (X31 and PR8). This work was supported by National Health and Medical Research Council of Australia to L.M.W.

\section{AUTHOR CONTRIBUTIONS}

L.M.W, I.C. and M.H.L designed the project. A.P. and L.M.W performed the experiments and data analysis. L.M.W, I.C. and M.H.L contributed to writing the manuscript.

\section{ADDITIONAL INFORMATION}

The online version of this article (https://doi.org/10.1038/s41385-018-0124-2) contains supplementary material, which is available to authorised users.

Conflict of interest: The authors declare that they have no conflict of interest.

Publisher's note: Springer Nature remains neutral with regard to jurisdictional claims in published maps and institutional affiliations.

\section{REFERENCES}

1. Pizzolla A, Nguyen THO, Smith JM, Brooks AG, Kedzieska K, Heath WR et al. Resident memory $\mathrm{CD} 8+\mathrm{T}$ cells in the upper respiratory tract prevent pulmonary influenza virus infection. Sci. Immunol. 2, pii: eaam6970 (2017). 
2. $W u, T$., Hu, Y., Lee, Y. T., Bouchard, K. R., Benechet, A. \& Khanna, K. et al. Lungresident memory CD8 $T$ cells (TRM) are indispensable for optimal cross-protection against pulmonary virus infection. J. Leukoc. Biol. 95, 215-24 (2014).

3. Wakim, L. M., Smith, J., Caminschi, I., Lahoud, M. H. \& Villadangos, J. A. Antibodytargeted vaccination to lung dendritic cells generates tissue-resident memory CD8 T cells that are highly protective against influenza virus infection. Mucosal Immunol. 8, 1060-71 (2015).

4. Turner DL, Bickham KL, Thome JJ, Kim CY, D'Ovidio F, Wherry EJ et al. Lung niches for the generation and maintenance of tissue-resident memory T cells. Mucosal Immunol. 7, 501-10 (2013).

5. Zens KD, Chen JK, Farber DL. Vaccine-generated lung tissue-resident memory $\mathrm{T}$ cells provide heterosubtypic protection to influenza infection. JCI Insight 1, pii: e85832 (2016).

6. Jozwik, A., Habibi, M. S., Paras, A., Zhu, J., Guvenel, A. \& Dhariwal, J. et al. RSVspecific airway resident memory CD8+T cells and differential disease severity after experimental human infection. Nat. Commun. 6, 10224 (2015).

7. Zhao, J., Zhao, J., Mangalam, A. K., Channappanavar, R., Fett, C. \& Meyerholz, D. K. et al. Airway memory $\mathrm{CD} 4(+) \mathrm{T}$ cells mediate protective immunity against emerging respiratory coronaviruses. Immunity 44, 1379-91 (2016).

8. Mueller, S. N. \& Mackay, L. K. Tissue-resident memory T cells: local specialists in immune defence. Nat. Rev. Immunol. 16, 79-89 (2016).

9. Mackay LK, Rahimpour A, Ma JZ, Collins N, Stock AT, Hafon ML et al. The developmental pathway for $\mathrm{CD} 103 \mathrm{CD} 8$ tissue-resident memory $\mathrm{T}$ cells of skin. Nat. Immunol.14, 1294-301 (2013).

10. Wakim, L. M., Woodward-Davis, A., Liu, R., Hu, Y., Villadangos, J. \& Smyth, G. et al. The molecular signature of tissue resident memory CD8 T cells isolated from the brain. J. Immunol. 189, 3462-71 (2012).

11. Hombrink, P., Helbig, C., Backer, R. A., Piet, B., Oja, A. E. \& Stark, R. et al. Programs for the persistence, vigilance and control of human CD8+lung-resident memory T cells. Nat. Immunol. 17, 1467-78 (2016).

12. Kumar, B. V., Ma, W., Miron, M., Granot, T., Guyer, R. S. \& Carpenter, D. J. et al. Human tissue-resident memory $T$ cells are defined by core transcriptional and functional signatures in lymphoid and mucosal sites. Cell Rep. 20, 2921-34 (2017).

13. Skon, C. N., Lee, J. Y., Anderson, K. G., Masopust, D., Hogquist, K. A. \& Jameson, S. C. Transcriptional downregulation of S1pr1 is required for the establishment of resident memory CD8+T cells. Nat. Immunol. 14, 1285-93 (2013).

14. Mackay, L. K., Wynne-Jones, E., Freestone, D., Pellicci, D. G., Mielke, L. A. \& Newman, D. M. et al. T-box transcription factors combine with the cytokines TGF-beta and IL-15 to control tissue-resident memory T cell fate. Immunity 43, 1101-11 (2015).

15. Mackay, L. K., Minnich, M., Kragten, N. A., Liao, Y., Nota, B. \& Seillet, C. et al. Hobit and Blimp1 instruct a universal transcriptional program of tissue residency in lymphocytes. Science 352, 459-63 (2016).

16. Ariotti, S., Hogenbirk, M. A., Dijkgraaf, F. E., Visser, L. L., Hoekstra, M. E. \& Song, J. Y. et al. T cell memory. Skin-resident memory CD8(+) T cells trigger a state of tissuewide pathogen alert. Science 346, 101-5 (2014).

17. Schenkel, J. M., Fraser, K. A., Vezys, V. \& Masopust, D. Sensing and alarm function of resident memory CD8(+) T cells. Nat. Immunol. 14, 509-13 (2013).

18. Hu, Y., Lee, Y. T., Kaech, S. M., Garvy, B. \& Cauley, L. S. Smad4 promotes differentiation of effector and circulating memory CD8 T cells but is dispensable for tissue-resident memory CD8 T cells. J. Immunol. 194, 2407-14 (2015).

19. Hu, Y. \& Cauley, L. Antigen and transforming growth factor Beta receptors contribute to long term functional and phenotypic heterogeneity of memory CD8 T cells. Front. Immunol. 4, 227 (2013).

20. Laidlaw, B. J., Zhang, N., Marshall, H. D., Staron, M. M., Guan, T. \& Hu, Y. et al. CD4 $+T$ cell help guides formation of $C D 103$ +lung-resident memory $C D 8+T$ cells during influenza viral infection. Immunity 41, 633-45 (2014).

21. Takamura, S., Yagi, H., Hakata, Y., Motozono, C., McMaster, S. R. \& Masumoto, T. et al. Specific niches for lung-resident memory $C D 8+T$ cells at the site of tissue regeneration enable CD69-independent maintenance. J. Exp. Med 213, 3057-73 (2016).

22. McMaster SR, Wein AN, Dunbar PR, Hayward SL, Cartwright EK, Denning TL et al. Pulmonary antigen encounter regulates the establishment of tissue-resident CD8 memory $\mathrm{T}$ cells in the lung airways and parenchyma. Mucosal Immunol. 11, 1071-1078 (2018).
23. Wakim, L. M., Gupta, N., Mintern, J. D. \& Villadangos, J. A. Enhanced survival of lung tissue-resident memory CD8 $(+)$ T cells during infection with influenza virus due to selective expression of IFITM3. Nat. Immunol. 14, 238-45 (2013).

24. Muschaweckh A, Buchholz VR, Fellenzer A, Hessel C, Konig PA, Tao S et al. Antigen-dependent competition shapes the local repertoire of tissue-resident memory CD8+T cells. J. Exp. Med. 213, 3075-3086 (2016).

25. Gebhardt, T., Wakim, L. M., Eidsmo, L., Reading, P. C., Heath, W. R. \& Carbone, F. R. Memory $T$ cells in nonlymphoid tissue that provide enhanced local immunity during infection with herpes simplex virus. Nat. Immunol. 10, 524-30 (2009).

26. Shin, H. \& Iwasaki, A. A vaccine strategy that protects against genital herpes by establishing local memory T cells. Nature 491, 463-7 (2012).

27. Bergsbaken, T. \& Bevan, M. J. Proinflammatory microenvironments within the intestine regulate the differentiation of tissue-resident $\mathrm{CD} 8(+) \mathrm{T}$ cells responding to infection. Nat. Immunol. 16, 406-14 (2015).

28. Gilchuk, P., Hill, T. M., Guy, C., McMaster, S. R., Boyd, K. L. \& Rabacal, W. A. et al. A distinct lung-interstitium-resident memory $\mathrm{CD} 8(+) \mathrm{T}$ cell subset confers enhanced protection to lower respiratory tract infection. Cell Rep. 16, 1800-9 (2016).

29. Deliyannis, G., Kedzierska, K., Lau, Y. F., Zeng, W., Turner, S. J. \& Jackson, D. C. et al. Intranasal lipopeptide primes lung-resident memory $C D 8+T$ cells for long-term pulmonary protection against influenza. Eur. J. Immunol. 36, 770-8 (2006).

30. Gasper, D. J., Neldner, B., Plisch, E. H., Rustom, H., Carrow, E. \& Imai, H. et al. Effective respiratory CD8 T-cell immunity to influenza virus induced by intranasal carbomer-lecithin-adjuvanted non-replicating vaccines. PLoS Pathog. 12, e1006064 (2016).

31. Underhill, D. M. Macrophage recognition of zymosan particles. J. Endotoxin Res. 9, 176-80 (2003).

32. Reid, D. M., Gow, N. A. \& Brown, G. D. Pattern recognition: recent insights from Dectin-1. Curr. Opin. Immunol. 21, 30-37 (2009).

33. Takeuchi, K., Umeki, Y., Matsumoto, N., Yamamoto, K., Yoshida, M. \& Suzuki, K. et al. Severe neutrophil-mediated lung inflammation in myeloperoxidasedeficient mice exposed to zymosan. Inflamm. Res. 61, 197-205 (2012).

34. Dillon, S., Agrawal, S., Banerjee, K., Letterio, J., Denning, T. L. \& Oswald-Richter, K. et al. Yeast zymosan, a stimulus for TLR2 and dectin-1, induces regulatory antigen-presenting cells and immunological tolerance. J. Clin. Invest. 116, 916-28 (2006).

35. Burton, O. T., Zaccone, P., Phillips, J. M., De La Pena, H., Fehervari, Z. \& Azuma, M. et al. Roles for TGF-beta and programmed cell death 1 ligand 1 in regulatory $T$ cell expansion and diabetes suppression by zymosan in nonobese diabetic mice. J. Immunol. 185, 2754-62 (2010).

36. Reis e Sousa, C., Stahl, P. D. \& Austyn, J. M. Phagocytosis of antigens by Langerhans cells in vitro. J. Exp. Med. 178, 509-19 (1993)

37. Samarasinghe, R., Tailor, P., Tamura, T., Kaisho, T., Akira, S. \& Ozato, K. Induction of an anti-inflammatory cytokine, IL-10, in dendritic cells after toll-like receptor signaling. J. Interferon Cytokine Res. 26, 893-900 (2006).

38. Saijo, K., Schmedt, C., Su, I. H., Karasuyama, H., Lowell, C. A. \& Reth, M. et al. Essential role of Src-family protein tyrosine kinases in NF-kappaB activation during B cell development. Nat. Immunol. 4, 274-9 (2003).

39. Goodridge, H. S., Simmons, R. M. \& Underhill, D. M. Dectin-1 stimulation by Candida albicans yeast or zymosan triggers NFAT activation in macrophages and dendritic cells. J. Immunol. 178, 3107-15 (2007).

40. Slack, E. C., Robinson, M. J., Hernanz-Falcon, P., Brown, G. D., Williams, D. L. \& Schweighoffer, E. et al. Syk-dependent ERK activation regulates IL-2 and IL-10 production by DC stimulated with zymosan. Eur. J. Immunol. 37, 1600-12 (2007).

41. Li, H., Gonnella, P., Safavi, F., Vessal, G., Nourbakhsh, B. \& Zhou, F. et al. Low dose zymosan ameliorates both chronic and relapsing experimental autoimmune encephalomyelitis. J. Neuroimmunol. 254, 28-38 (2013).

42. Wakim, L. M., Woodward-Davis, A. \& Bevan, M. J. Memory T cells persisting within the brain after local infection show functional adaptations to their tissue of residence. Proc. Natl. Acad. Sci. USA 107, 17872-9 (2010).

43. Li, X., Liu, Z., Jin, H., Fan, X., Yang, X. \& Tang, W. et al. Agmatine protects against zymosan-induced acute lung injury in mice by inhibiting NF-kappaB-mediated inflammatory response. Biomed. Res. Int. 2014, 583736 (2014). 